\title{
Dynamic Electromechanical Response of a Viscoelastic Dielectric Elastomer under Cycle Electric Loads
}

\author{
Junjie Sheng $\mathbb{D}$ and Yuqing Zhang \\ Institute of Systems Engineering, China Academy of Engineering Physics, Mianyang 621999, China \\ Correspondence should be addressed to Junjie Sheng; scu2005sjj@163.com
}

Received 8 October 2017; Accepted 28 December 2017; Published 29 January 2018

Academic Editor: Jianwen Zhao

Copyright (C) 2018 Junjie Sheng and Yuqing Zhang. This is an open access article distributed under the Creative Commons Attribution License, which permits unrestricted use, distribution, and reproduction in any medium, provided the original work is properly cited.

\begin{abstract}
Dielectric elastomer (DE) is able to produce large electromechanical deformation which is time-dependent due to the viscoelasticity. In the current study, a thermodynamic model is set up to characterize the influence of viscoelasticity on the electromechanical and dynamic response of a viscoelastic DE. The time-dependent dynamic deformation, the hysteresis, and the dynamic stability undergoing viscoelastic dissipative processes are investigated. The results show that the electromechanical stability has strong frequency dependence; the viscoelastic DE can attain a larger stretch in the dynamic response than the quasistatic actuation. Furthermore, with the decreasing frequency of the applied electric load, the viscoelastic DE system will present dynamic stability evolution from an aperiodic motion to the quasiperiodic motion. The DE system may also experience a stability evolution from a single cycle motion to multicycle motion with the increasing relaxation times. The value and variation trend of the amplitude of the stretch are highly dependent on the excitation frequency and the relaxation time.
\end{abstract}

\section{Introduction}

Due to their fast response time, soft, lightweight, low-cost, and high energy density, dielectric elastomers (DEs) have been developed to use in high performance applications as artificial muscles, Braille displays, life-like robots, tunable lens, and power generators [1-6]. It consists of a soft elastomeric membrane sandwiched between two compliant electrodes on both sides. Most of the existing studies on DEs have focused on quasistatic deformation [5-8], neglecting the effect of inertia and viscoelasticity.

However, to perform as an electromechanical actuator, a Dielectric elastomer (DE) is often subject to transient, timedependent forces and voltages [9-11] and is mostly expected to deform at high frequencies in applications, where inertia and viscoelasticity can play a significant role in the dynamic application. Applications exploiting the dynamic behavior of DEs have long been realized through experiments including vibrotactile display for mobile applications [12], frequency tuning [13], pumps [14], and acoustic actuator [15]. Recently, researches have been also carried out on modeling the nonlinear vibrations of hyperelastic DE membranes [16-19]. Zhu et al. [16] studied the resonant behavior of a prestretched membrane of a DE and subsequently analyzed the nonlinear oscillations of a DE balloon [17]. Based on simple geometrical and spherical capacitor assumptions, Yong et al. [18] investigated the dynamics of a thick-walled DE spherical shell. Li et al. [19] analyzed the electromechanical and dynamic analyses of tunable pure-shear DE-based resonator and identified the safe operation range for failure prevention while actuating the resonator. $\mathrm{Xu}$ et al. [20] obtained an analytical model for the DE by the Euler-Lagrange equation to study the dynamic analysis of a DE with stretching deformation. Jia et al. [21] investigated the response time and dynamic range for a $\mathrm{DE}$ actuator. Li et al. [22] presented an analysis of the nonlinear dynamics of a DE as electromechanical resonator (DEER) configured as a pure-shear actuator. An investigation in our previous paper [23] reported the nonlinear dynamic characteristics of a DE membrane undergoing in-plane deformation. Wang et al. [24] investigated the strain-stiffening effect on the nonlinear vibration of a circular DE membrane subjected to electromechanical load. 
Although the above studies have attempted to model the nonlinear dynamical behavior of the DE to probe its timedependent performance, they have neglected the viscoelasticity of DE. The time-dependency of a DE can cause dissipation in the system and significantly affect its dynamic performance and coupling efficiency $[25,26]$. In particular, experiments have shown that viscoelasticity can significantly influence the electromechanical transduction and its application $[4,9,10$, 25-27].

Recently, a few studies have been developed concerning the dynamic performance of viscoelastic DE [28-32]. Zhang et al. [28] studied the coupled nonlinear oscillation and stability evolution of viscoelastic DE under nonequibiaxial tensile forces by utilizing the method of virtual work, developed an analytical model to characterize the dynamic performance of a homogeneously deformed viscoelastic DE under the conditions of equal-biaxial force, uniaxial force, and pureshear state by using the Euler-Lagrange equation [29], and predicted the damping effect on the dynamic performance of DE based on the standard linear solid rheological model [30]. Wang et al. [31] presented a model describing the nonlinear dynamic visco-hyperelastic behaviors of DE and explained the material's dynamic energy dissipation mechanism. However, the effect of viscoelasticity and relaxation time on hysteresis and stability evolution of the DE was neglected in previously reported papers. Zhang et al. [32] investigated the static and dynamic performance of a hinge configuration with integrated dielectric elastomers. Our previous paper [33] also investigated the effect of temperature on the dynamic electromechanical performance of viscoelastic dielectric.

Based on the theory of nonequilibrium thermodynamics [34-36] and the nonlinear vibration modeling [16-19], we aim to characterize the electromechanical and dynamic response of viscoelastic dielectric elastomer, to predict how viscoelasticity affect its dynamic performance and hysteresis process by comparing with the quasistatic response, and to present a physical interpretation on the instability and stability evolution coupled by viscoelasticity, relaxation time, and dynamic deformation.

In this study, the influence of the viscoelasticity effects on the electromechanical and dynamic characteristics of a DE membrane is considered by setting up a thermodynamic model. With the proposed model, the dynamic responses of a DE film subject to a cyclic electric field are investigated and compared with the quasistatic response. Then, the dynamic oscillation, phase diagrams, and Poincaré maps of the viscoelastic DE are studied. A detailed analysis shows the influence of the frequency, viscoelasticity, and relaxation time on the dynamic stability evolution and the hysteresis.

\section{Thermodynamic Modeling of a Viscoelastic DE}

Considering a piece of viscoelastic dielectric, it deforms when subject to both in-plane biaxial force and a voltage-induced force through the thickness. Figure 1 illustrates a membrane of a DE, sandwiched between two compliant electrodes. The $\mathrm{DE}$ deforms from its original configuration $L_{1} \times L_{2} \times L_{3}$ to the current configuration $l_{1} \times l_{2} \times l_{3}$ in terms of an equal biaxial expansion. Here, we define $\lambda_{i}(i=1,2,3)=l_{i} / L_{i}$ as the stretch ratio. The stretch $\lambda_{i}$ is homogeneous through the $\mathrm{DE}$ and is a function of time. Due to the incompressibility of the material, we express the stretch ratio in the thickness direction as $\lambda_{3}=\lambda_{1}^{-1} \lambda_{2}^{-1}$.

The nominal stresses are defined as $s_{1}=P_{1} /\left(L_{2} L_{3}\right)$ and $s_{2}=P_{2} /\left(L_{1} L_{3}\right)$. The nominal electric field is $\widetilde{E}=\Phi / L_{3}$ and the nominal electric displacement is defined by $\widetilde{D}=Q / L_{1} L_{2}$, the true electric field by $E=\Phi / l_{3}$, and the true electric displacement by $D=Q /\left(l_{1} l_{2}\right)$. We adopt the model of ideal $\mathrm{DE}$, assuming that the permittivity of the elastomer $\varepsilon$ is taken to be constant. That is, the true electric displacement is taken to be linear in the true electric field, $D=\varepsilon E[19,33,34]$. Based on the assumption of the DE membrane as a parallel capacitor, we obtain the relation between the charge and the voltage as

$$
Q=\Phi \frac{\varepsilon L_{1} L_{2}}{L_{3}} \lambda_{1}^{2} \lambda_{2}^{2}
$$

When the membrane is subject to forces and voltage, all three quantities $\Phi, \lambda_{1}$, and $\lambda_{2}$ can vary, so that the variation of the charge is

$$
\begin{aligned}
\delta Q= & \frac{\varepsilon L_{1} L_{2}}{L_{3}} \lambda_{1}^{2} \lambda_{2}^{2} \delta \Phi \\
& +\Phi \frac{\varepsilon L_{1} L_{2}}{L_{3}}\left(2 \lambda_{1} \lambda_{2}^{2} \delta \lambda_{1}+2 \lambda_{2} \lambda_{1}^{2} \delta \lambda_{2}\right) .
\end{aligned}
$$

When the dimensions of the DE varies slightly by $\delta \lambda_{1}$ and $\delta \lambda_{2}$, the tensile forces do work of $P_{1} L_{1} \delta \lambda_{1}+P_{2} L_{2} \delta \lambda_{2}$. Associated with a small amount of charge $\delta Q$ moving through the conducting wire, the applied voltage does a work of $\Phi \delta Q$. During actuation, the inertia forces in each material element along the $x$-direction and $y$-direction are $\rho L_{2} L_{3} x^{2}\left(\mathrm{~d}^{2} \lambda_{1} / \mathrm{d} t^{2}\right)$ and $\rho L_{1} L_{3} y^{2}\left(\mathrm{~d}^{2} \lambda_{2} / \mathrm{d} t^{2}\right)$, respectively [19]. The damping forces along the $x$-direction and $y$-direction are $c x \mathrm{~d} \lambda_{1} / \mathrm{d} t$ and $c y \mathrm{~d} \lambda_{2} / \mathrm{d} t$, respectively, which are linear with respect to the deformation velocity in these directions, where $\rho$ is the density of the elastomer and $c$ is the damping coefficient [20]. By its displacement derivative along the $x$-direction and $y$ direction, it is not difficult to obtain the inertial force does work and the damping force does work as

$$
\begin{aligned}
\rho L_{2} L_{3} \frac{\mathrm{d}^{2} \lambda_{1}}{\mathrm{~d} t^{2}} \delta \lambda_{1} \int_{0}^{L_{1}} x^{2} \mathrm{~d} x & =\frac{L_{1}^{3} \rho L_{2} L_{3}}{3} \frac{\mathrm{d}^{2} \lambda_{1}}{\mathrm{~d} t^{2}} \delta \lambda_{1} \\
\rho L_{1} L_{3} \frac{\mathrm{d}^{2} \lambda_{2}}{\mathrm{~d} t^{2}} \delta \lambda_{2} \int_{0}^{L_{2}} y^{2} \mathrm{~d} y & =\frac{L_{2}^{3} \rho L_{1} L_{3}}{3} \frac{\mathrm{d}^{2} \lambda_{2}}{\mathrm{~d} t^{2}} \delta \lambda_{2} \\
c \frac{\mathrm{d} \lambda_{1}}{\mathrm{~d} t} \delta \lambda_{1} \int_{0}^{L_{1}} x \mathrm{~d} x & =\frac{1}{2} c L_{1}^{2} \delta \lambda_{1} \frac{\mathrm{d} \lambda_{1}}{\mathrm{~d} t} \\
c \frac{\mathrm{d} \lambda_{2}}{\mathrm{~d} t} \delta \lambda_{2} \int_{0}^{L_{2}} y \mathrm{~d} x & =\frac{1}{2} c L_{2}^{2} \delta \lambda_{2} \frac{\mathrm{d} \lambda_{2}}{\mathrm{~d} t} .
\end{aligned}
$$

Thermodynamics requires that, for arbitrary variation of the system, the variation of the free energy of the membrane 


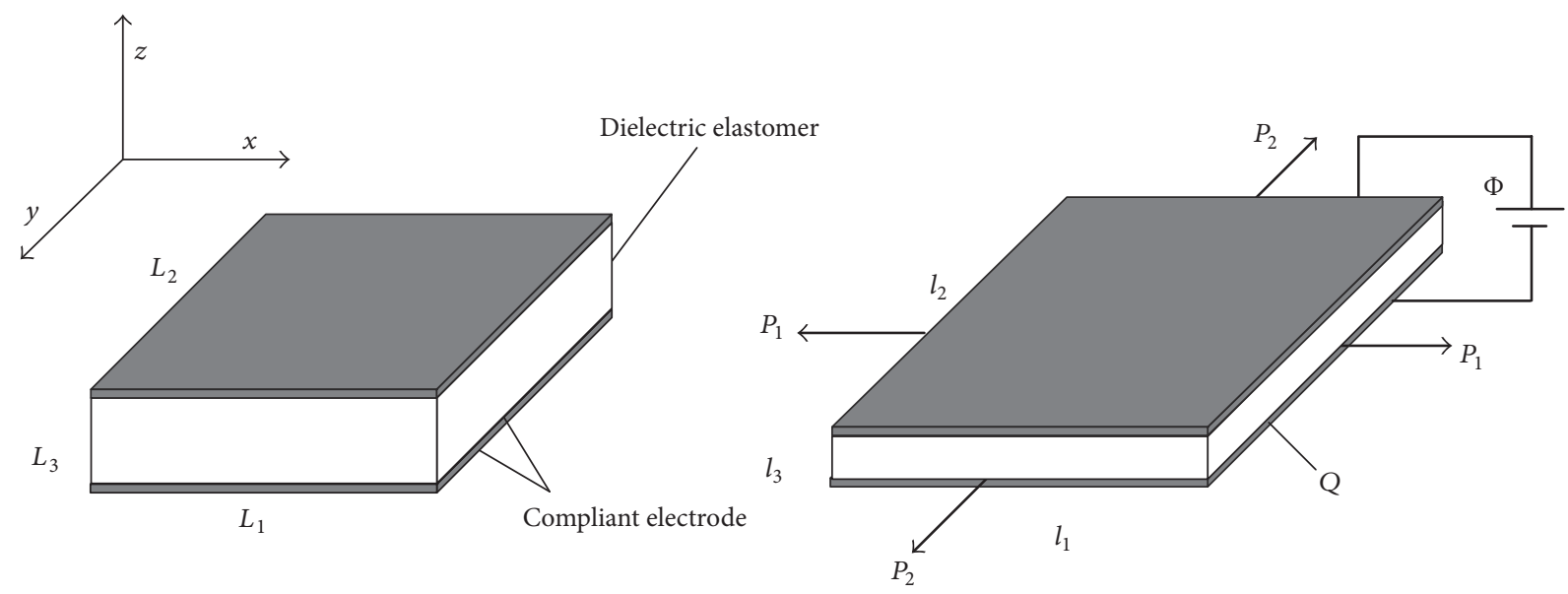

Figure 1: Schematics of a viscoelastic dielectric elastomer subject biaxial force in the plane and voltage through the thickness applied via stretchable electrodes.

should equal the work done by the voltage, the prestress, the inertia force, and the damping force; namely,

$$
\begin{aligned}
L_{1} L_{2} L_{3} \delta W= & \Phi \delta Q+P_{1} L_{1} \delta \lambda_{1}+P_{2} L_{2} \delta \lambda_{2} \\
& -\frac{L_{1}^{3} \rho L_{2} L_{3}}{3} \frac{\mathrm{d}^{2} \lambda_{1}}{\mathrm{~d} t^{2}} \delta \lambda_{1} \\
& -\frac{L_{2}^{3} \rho L_{1} L_{3}}{3} \frac{\mathrm{d}^{2} \lambda_{2}}{\mathrm{~d} t^{2}} \delta \lambda_{2}-\frac{1}{2} c L_{1}^{2} \delta \lambda_{1} \frac{\mathrm{d} \lambda_{1}}{\mathrm{~d} t} \\
& -\frac{1}{2} c L_{2}^{2} \delta \lambda_{2} \frac{\mathrm{d} \lambda_{2}}{\mathrm{~d} t} .
\end{aligned}
$$

Experiments have verified that the electromechanical responses of DEs are highly rate-dependent, which implies that the deformation and actuation of DEs are highly relying on the rates of mechanical or electrical activation $[9,10]$. This rate-dependence is mainly induced by the viscoelasticity of the elastomeric polymer matrix and may consequently influence the electromechanical actuation [27]. Viscoelastic relaxation may be represented by a rheological model of springs and dashpots [34-36]. As the DE exhibits elastic deformation and inelastic deformation, the latter of which is time-dependent, we first model this material by assuming that it is composed of two molecular chain networks, A and B, as sketched in Figure 2. The network A is an ideal hyperelastic chain and deforms reversibly, while the network B relaxes in time and dissipates energy. The viscous deformation is represented by a dashpot.

For the network represented by the spring and the dashpot at the bottom, the stretches $\left(\lambda_{1}, \lambda_{2}\right)$ are due to both the spring and the dashpot; we adopt the well-established multiplication rule that [35-37]

$$
\begin{aligned}
& \lambda_{1}=\lambda_{1}^{e} \xi_{1}, \\
& \lambda_{2}=\lambda_{2}^{e} \xi_{2},
\end{aligned}
$$

where $\left(\lambda_{1}^{e}, \lambda_{2}^{e}\right)$ are the stretches due to the bottom spring and $\left(\xi_{1}, \xi_{2}\right)$ are stretches due to the dashpot.

To account for the effect of strain-stiffening, we represent both springs by using the Gent model [36-38]. The freeenergy function of the elastomer is the sum of the contributions from the two springs and can be written as

$W$

$$
\begin{aligned}
= & -\frac{\mu^{\mathrm{A}} J^{\mathrm{A}}}{2} \ln \left(1-\frac{\lambda_{1}^{2}+\lambda_{2}^{2}+\lambda_{1}^{-2} \lambda_{2}^{-2}-3}{J^{\mathrm{A}}}\right) \\
& -\frac{\mu^{\mathrm{B}} J^{\mathrm{B}}}{2} \ln \left(1-\frac{\lambda_{1}^{2} \xi_{1}^{-2}+\lambda_{2}^{2} \xi_{2}^{-2}+\lambda_{1}^{-2} \lambda_{2}^{-2} \xi_{1}^{2} \xi_{2}^{2}-3}{J^{\mathrm{B}}}\right) \\
& +\frac{\varepsilon}{2}\left(\frac{\Phi}{L_{3}}\right)^{2} \lambda_{1}^{2} \lambda_{2}^{2},
\end{aligned}
$$

where $\mu^{\mathrm{A}}$ and $\mu^{\mathrm{B}}$ are the shear moduli of the two springs and $J^{\mathrm{A}}$ and $J^{\mathrm{A}}$ are the extension limits.

A combination of (2) and (4) gives that

$$
\begin{aligned}
L_{1} L_{2} L_{3} \delta W= & \Phi^{2} \frac{\varepsilon L_{1} L_{2}}{L_{3}}\left(2 \lambda_{1} \lambda_{2}^{2} \delta \lambda_{1}+2 \lambda_{1}^{2} \lambda_{2} \delta \lambda_{2}\right) \\
& +\Phi \frac{\varepsilon L_{1} L_{2}}{L_{3}} \lambda_{1}^{2} \lambda_{2}^{2} \delta \Phi+P_{1} L_{1} \delta \lambda_{1} \\
& +P_{2} L_{2} \delta \lambda_{2}-\frac{L_{1}^{3} \rho L_{2} L_{3}}{3} \frac{\mathrm{d}^{2} \lambda_{1}}{\mathrm{~d} t^{2}} \delta \lambda_{1} \\
& -\frac{L_{2}^{3} \rho L_{1} L_{3}}{3} \frac{\mathrm{d}^{2} \lambda_{2}}{\mathrm{~d} t^{2}} \delta \lambda_{2}-\frac{1}{2} c L_{1}^{2} \delta \lambda_{1} \frac{\mathrm{d} \lambda_{1}}{\mathrm{~d} t} \\
& -\frac{1}{2} c L_{2}^{2} \delta \lambda_{2} \frac{\mathrm{d} \lambda_{2}}{\mathrm{~d} t} .
\end{aligned}
$$




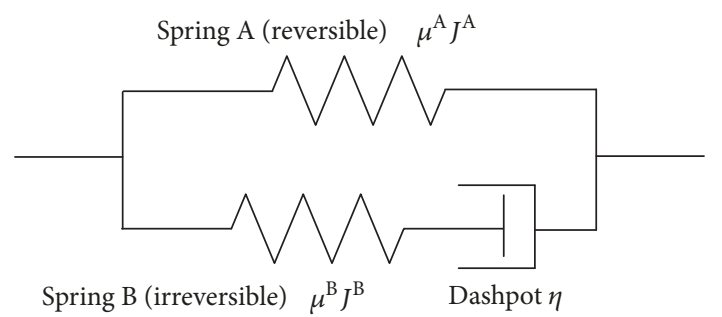

FIgURE 2: A Schematic illustration of a viscoelastic model for dielectric elastomer. It consists of two parallel elements: one element is a spring $\mathrm{A}$ and the other is a spring $\mathrm{B}$ which connected a dashpot in series.

We assume that the system is in mechanical and electrostatic equilibrium, and we obtain from the standard calculus of variation in (7) that

$$
\begin{aligned}
\frac{\partial W}{\partial \lambda_{1}}= & 2 \varepsilon\left(\frac{\Phi}{L_{3}}\right)^{2} \lambda_{1} \lambda_{2}^{2}+\frac{P_{1}}{L_{2} L_{3}}-\frac{L_{1}^{2} \rho}{3} \frac{\mathrm{d}^{2} \lambda_{1}}{\mathrm{~d} t^{2}} \\
& -\frac{1}{2} \frac{c L_{1}}{L_{2} L_{3}} \frac{\mathrm{d} \lambda_{1}}{\mathrm{~d} t}, \\
\frac{\partial W}{\partial \lambda_{2}}= & 2 \varepsilon\left(\frac{\Phi}{L_{3}}\right)^{2} \lambda_{1}^{2} \lambda_{2}+\frac{P_{2}}{L_{1} L_{3}}-\frac{L_{2}^{2} \rho}{3} \frac{\mathrm{d}^{2} \lambda_{2}}{\mathrm{~d} t^{2}} \\
& -\frac{1}{2} \frac{c L_{2}}{L_{1} L_{3}} \frac{\mathrm{d} \lambda_{2}}{\mathrm{~d} t}, \\
\widetilde{D}= & \frac{\partial W}{\partial \widetilde{E}}=\Phi \frac{\varepsilon}{L_{3}} \lambda_{1}^{2} \lambda_{2}^{2}=\varepsilon \widetilde{E} \lambda_{1}^{2} \lambda_{2}^{2} .
\end{aligned}
$$

Inserting (6) into (8) and (9), we obtain that

$$
\begin{gathered}
\frac{L_{1}^{2} \rho}{3} \frac{\mathrm{d}^{2} \lambda_{1}}{\mathrm{~d} t^{2}}+\frac{\mu^{\mathrm{A}}\left(\lambda_{1}-\lambda_{1}^{-3} \lambda_{2}^{-2}\right)}{1-\left(\lambda_{1}^{2}+\lambda_{2}^{2}+\lambda_{1}^{-2} \lambda_{2}^{-2}-3\right) / J^{\mathrm{A}}} \\
+\frac{\mu^{\mathrm{B}}\left(\lambda_{1} \xi_{1}^{-2}-\lambda_{1}^{-3} \lambda_{2}^{-2} \xi_{1}^{2} \xi_{2}^{2}\right)}{1-\left(\lambda_{1}^{2} \xi_{1}^{-2}+\lambda_{2}^{2} \xi_{2}^{-2}+\lambda_{1}^{-2} \lambda_{2}^{-2} \xi_{1}^{2} \xi_{2}^{2}-3\right) / J^{\mathrm{B}}} \\
-\varepsilon\left(\frac{\Phi}{L_{3}}\right)^{2} \lambda_{1} \lambda_{2}^{2}-\frac{P_{1}}{L_{2} L_{3}}+\frac{1}{2} \frac{c L_{1}}{L_{2} L_{3}} \frac{\mathrm{d} \lambda_{1}}{\mathrm{~d} t}=0, \\
\frac{L_{2}^{2} \rho}{3} \frac{\mathrm{d}^{\mathrm{A}} \lambda_{2}}{\mathrm{~d} t^{2}}+\frac{\left.\mu_{2}-\lambda_{1}^{-2} \lambda_{2}^{-3}\right)}{1-\left(\lambda_{1}^{2}+\lambda_{2}^{2}+\lambda_{1}^{-2} \lambda_{2}^{-2}-3\right) / J^{\mathrm{A}}} \\
+\frac{\mu^{\mathrm{B}}\left(\lambda_{2} \xi_{2}^{-2}-\lambda_{1}^{-2} \lambda_{2}^{-3} \xi_{1}^{2} \xi_{2}^{2}\right)}{1-\left(\lambda_{1}^{2} \xi_{1}^{-2}+\lambda_{2}^{2} \xi_{2}^{-2}+\lambda_{1}^{-2} \lambda_{2}^{-2} \xi_{1}^{2} \xi_{2}^{2}-3\right) / J^{\mathrm{B}}} \\
-\varepsilon\left(\frac{\Phi}{L_{3}}\right)^{2} \lambda_{1}^{2} \lambda_{2}-\frac{P_{2}}{L_{2} L_{3}}+\frac{1}{2} \frac{c L_{2}}{L_{1} L_{3}} \frac{\mathrm{d} \lambda_{2}}{\mathrm{~d} t}=0 .
\end{gathered}
$$

To simplify mathematical expressions, we introduce the following dimensionless field variables $s_{1}=P_{1} /\left(L_{2} L_{3} \mu\right)$, $\bar{E}=\Phi /\left(L_{3} \sqrt{\varepsilon / \mu}\right)=\widetilde{E} / \sqrt{\varepsilon / \mu}, s_{1}=P_{2} /\left(L_{1} L_{3} \mu\right)$, and $\bar{D}=$ $Q /\left(L_{1} L_{2} \sqrt{\varepsilon \mu}\right)=\widetilde{D} / \sqrt{\varepsilon \mu}$.
While the method described here is applicable to general cases, in the following discussion, we will look at a special case when the dielectric film is under equibiaxial stress, such that $L_{1}=L_{2}=L, \lambda_{1}=\lambda_{2}=\lambda, \xi_{1}=\xi_{2}=\xi$, and $s_{1}=$ $s_{2}=s$. The symmetry of the problem reduces the governing equations (11) to

$$
\begin{aligned}
& \frac{\rho L^{2}}{3 \mu} \frac{\mathrm{d}^{2} \lambda}{\mathrm{d} t^{2}}+\frac{\chi\left(\lambda-\lambda^{-5}\right)}{1-\left(2 \lambda^{2}+\lambda^{-4}-3\right) / J^{\mathrm{A}}} \\
& \quad+\frac{(1-\chi)\left(\lambda \xi^{-2}-\lambda^{-5} \xi^{4}\right)}{1-\left(2 \lambda^{2} \xi^{-2}+\lambda^{4} \xi^{4}-3\right) / J^{\mathrm{B}}}-\frac{\varepsilon}{\mu}\left(\frac{\Phi}{L_{3}}\right)^{2} \lambda^{3} \\
& \quad-s+\frac{1}{2} \frac{c}{\mu L_{3}} \frac{\mathrm{d} \lambda}{\mathrm{d} T}=0,
\end{aligned}
$$

where $\mu=\mu^{\mathrm{A}}+\mu^{\mathrm{B}}$ and $\chi=\mu^{\mathrm{A}} / \mu$ are the ratio between the equilibrium and instantaneous moduli. The rate of deformation in the dashpot is described by $\xi^{-1} \mathrm{~d} \xi / \mathrm{d} t$. We relate the rate of deformation in the dashpot to the stress on the dashpot and write as [36]

$$
\frac{\mathrm{d} \xi}{\mathrm{d} t}=\frac{\xi \mu^{\mathrm{B}}}{6 \eta} \frac{\left(\lambda^{2} \xi^{-2}-\lambda^{-4} \xi^{4}\right)}{1-\left(2 \lambda^{2} \xi^{-2}+\lambda^{-4} \xi^{4}-3\right) / J^{\mathrm{B}}},
$$

where $\eta$ is the viscosity of the dashpot.

We can see that the qualitative properties of the solutions of (12) and (13) depend on the applied loads and viscoelasticity. Equations (12) and (13) give the equations of motion for a viscoelastic DE, which we use in the following analysis to study the quasistatic response and the dynamic characteristics of a viscoelastic DE.

\section{Simulation Results and Discussion}

3.1. Quasistatic Response. When the stress $s$ and voltage $\Phi$ are quasistatic, the membrane may reach a state of equilibrium. In this case, both the inertial force and the viscous force are zero; in the state of equilibrium, the equation of motion (12) can be expressed as

$$
\begin{aligned}
& \frac{\chi\left(\lambda-\lambda^{-5}\right)}{1-\left(2 \lambda^{2}+\lambda^{-4}-3\right) / J^{\mathrm{A}}} \\
& \quad+\frac{(1-\chi)\left(\lambda \xi^{-2}-\lambda^{-5} \xi^{4}\right)}{1-\left(2 \lambda^{2} \xi^{-2}+\lambda^{4} \xi^{4}-3\right) / J^{\mathrm{B}}}-\bar{E}^{2} \lambda^{3}-s=0 .
\end{aligned}
$$

The inelastic deformation of the material evolves with relaxation time $\eta / \mu^{\mathrm{B}}$. Here we will use it to normalize time $t$ by introducing a dimensionless time, $\tau=t \mu^{\mathrm{B}} / \eta$. Using the dimensionless time, we write the kinetic equation (13) as

$$
\begin{aligned}
& \frac{\chi\left(\lambda-\lambda^{-5}\right)}{1-\left(2 \lambda^{2}+\lambda^{-4}-3\right) / J^{\mathrm{A}}} \\
& \quad+\frac{(1-\chi)\left(\lambda \xi^{-2}-\lambda^{-5} \xi^{4}\right)}{1-\left(2 \lambda^{2} \xi^{-2}+\lambda^{4} \xi^{4}-3\right) / J^{\mathrm{B}}}-\bar{E}^{2} \lambda^{3}-s=0 .
\end{aligned}
$$


In the following analysis, we take $J^{\mathrm{A}}=110 \mathrm{kPa}, J^{\mathrm{B}}=$ $55 \mathrm{kPa}, \chi=0.5$ [36] as representative values for the most widely used $\mathrm{DE}$.

When the viscoelastic DE actuator subject to a cyclic electric load, $\bar{E}=\bar{E}_{0} \sin (\omega \tau)$, but not to forces $s=0$, with $\bar{E}_{0}=0.5$ to avoid instantaneous instability within the selected frequency range and with $\omega$ being the dimensionless frequency, the total stretch $\lambda$ and the inelastic stretch $\xi$ can be easily evolved by solving (14) and (15). The voltage is applied at time $\tau=0$. The dashpot does not move instantaneously, so that the initial value of the internal variable is $\lambda=\xi=1$. The time-dependent stretch, nominal electric field-stretch, and nominal electric field-nominal electric displacement curves of representative applied voltage frequency are presented in Figure 3.

It can be seen that, at a relatively high frequency $(\omega=10)$ of the applied electric field, the deformation of the viscoelastic DE could keep in phase with the electric field, as shown in Figure 3(a), but the actuation strain $(\lambda)$ is relatively small (shown with solid curves in Figure 3(a)). This is because that the voltage was varied on a time-scale much faster than the viscoelastic relaxation time. Subjecting to a cyclic load, as the actuation stretch only depends on the magnitude of the applied voltage, the frequency of the stretch doubles that of the electric field in Figure 3(a), and the mean value of stretch may drift away from the initial equilibrium state. The nominal electric field-stretch response $(\bar{E}-\lambda)$ in Figure 3(b) and the nominal electric field-nominal electric displacement response $(\bar{E}-\bar{D})$ in Figure 3(c) show no clear limit cycle, indicating that there will be insignificant hysteresis in the viscoelastic $\mathrm{DE}$ for high frequency.

When the frequency of the applied electric field decreases to an intermediate frequency, for example, $\omega=0.1$, the magnitude of the actuation strain $(\lambda)$ becomes much larger, and the amplitude of the inelastic stretch is almost the same as that of $\lambda$, showing almost fully inelastic deformation. Limit cycles appear on $\bar{E}-\lambda$ and $\bar{E}-\bar{D}$ plots in Figures 3(e) and $3(\mathrm{f})$. Both plots show hysteresis which indicate that significant amount of energy is dissipated during the electromechanical conversion process.

When the DE is actuated by a relatively low frequency voltage for $\omega=0.034$, the magnitude of both the actuation stretch and the inelastic stretch attains peak values and there will be most dissipative energy during this cycle, as shown in Figures $3(\mathrm{~g}), 3(\mathrm{~h})$, and 3(i). The main reason is the geometric nonlinearity introduced by the finite viscoelastic deformation in (14) and (15) according to Hong [34]. When the input electric field is sinusoidal, the output total stretch $(\lambda)$ and the inelastic stretch $(\xi)$ are not sinusoidal any more. The reason is that the thermoelectromechanically system under a cycle electric load is highly nonlinear and the asymmetry in the shape of the output cyclic deformation $\lambda(\tau)$ at low frequency would lead to significant irreversible energy loss of the system.

However, when the frequency decrease to a critical value, for $\omega \leq 0.033$, the viscoelastic DE will suffer the pull-in instability a little while as plotted in Figures $3(j), 3(k)$, and $3(1)$, the instability is marked with " $\times$." When the voltage is applied at a relatively low frequency, the major part of the total deformation is inelastic as shown in Figure 3(j). This result agrees well with experimental observations [9] that the DE more often suffers the pull-in instability at low stretch rates when inelastic deformation is significant.

3.2. Dynamic Response. When the DE with stretching deformation oscillates under cycle voltage, the inertia force will contribute to the electromechanical performance. In the following, numerical simulation results of the model are presented to show the vibration and oscillation behavior of the viscoelastic DE.

Here we will use the normalized time $t$ by introducing the dimensionless time $\tilde{t}=t /(L \sqrt{\rho / 3 \mu}), \widetilde{c}=c /\left(2 \mu L_{3} L \sqrt{\rho / 3 \mu}\right)$ and dimensionless relaxation time $\tilde{\tau}=\left(\eta / \mu^{\mathrm{B}}\right) /(L \sqrt{\rho / 3 \mu})$, and we may reduce (12) and (13) to the following form:

$$
\begin{aligned}
& \frac{\mathrm{d}^{2} \lambda}{\mathrm{d} \widetilde{t}^{2}}+\frac{\chi\left(\lambda-\lambda^{-5}\right)}{1-\left(2 \lambda^{2}+\lambda^{-4}-3\right) / J^{\mathrm{A}}} \\
& \quad+\frac{(1-\chi)\left(\lambda \xi^{-2}-\lambda^{-5} \xi^{4}\right)}{1-\left(2 \lambda^{2} \xi^{-2}+\lambda^{4} \xi^{4}-3\right) / J^{\mathrm{B}}}-\bar{E}^{2} \lambda^{3}-s \\
& \quad+\widetilde{c} \frac{\mathrm{d} \lambda}{\mathrm{d} T}=0, \\
& \frac{\mathrm{d} \xi}{\mathrm{d} \widetilde{t}}=\frac{1}{6 \widetilde{\tau}} \frac{\xi\left(\lambda^{2} \xi^{-2}-\lambda^{-4} \xi^{4}\right)}{1-\left(2 \lambda^{2} \xi^{-2}+\lambda^{-4} \xi^{4}-3\right) / J^{\mathrm{B}}} .
\end{aligned}
$$

To be specific, the following parameters are used in the dynamic examples: $J^{\mathrm{A}}=110 \mathrm{KPa}, J^{\mathrm{B}}=55 \mathrm{KPa}, \chi=0.5$, $\widetilde{\tau}=1$, and $\widetilde{c}=1[20,34-36]$.

The cyclic electric load, $\bar{E}=\bar{E}_{0} \sin (\Omega \widetilde{t})$, is applied at time $\tilde{t}=0$, with $\bar{E}_{0}=0.5$ to avoid instantaneous instability within the selected frequency range and with $\Omega$ being the dimensionless frequency. The dashpot does not move instantaneously, so that the initial value of the internal variable is $\lambda(0)=\xi(0)=1$ and $\dot{\lambda}(0)=0$. Under these assumptions, when no prestress is applied, $s=0$, by solving the governing equations (16), the dynamic stability evolution and performance of the viscoelastic elastomer, such as the oscillation diagram, the hysteresis loop, the phase diagram, and the Poincare map, are simulated.

Due to nonlinearity of the system, the dynamic response of the viscoelastic elastomer under time-dependent electric load can be very complicated. When the representative dimensionless frequency varies from 0 to 10 , the viscoelastic DE presents oscillations with varying amplitudes shown in Figures 4-7.

It can be seen that voltage applied at a relative high frequency $(\Omega=10)$ gives a relative small total stretch $(\lambda)$ and viscous stretch $(\xi)$ in Figure 4(a). At the same time, the nominal electric field-stretch response $(\bar{E}-\lambda)$ in Figure $4(\mathrm{~b})$ and the nominal electric field-nominal electric displacement response $(\bar{E}-\bar{D})$ in Figure 4(c) show no clear limit cycle, indicating that there will be insignificant hysteresis in the 

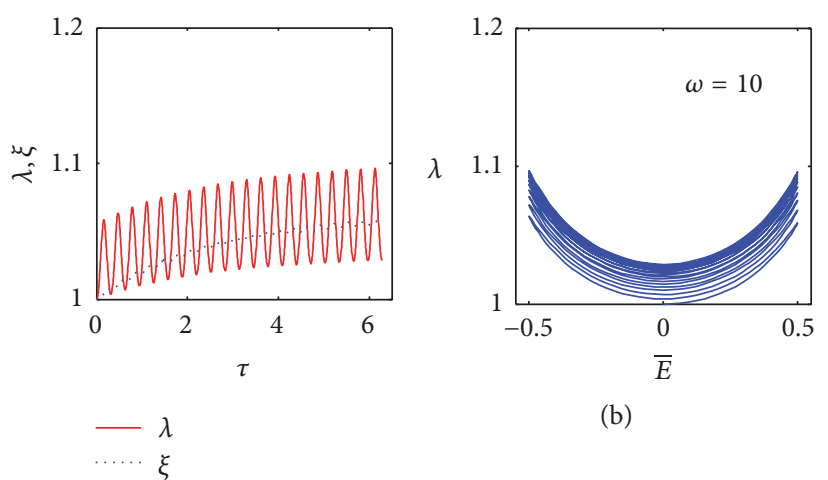

(b)

(a)

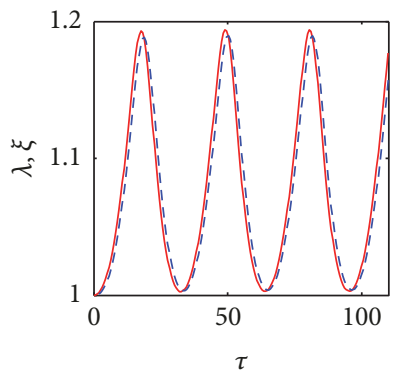

$\begin{array}{rr}- & \lambda \\ --- & \xi\end{array}$

(d)
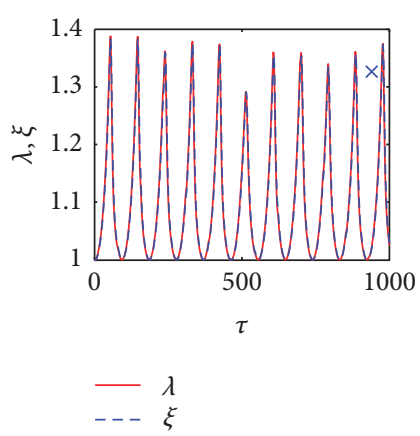

(g)

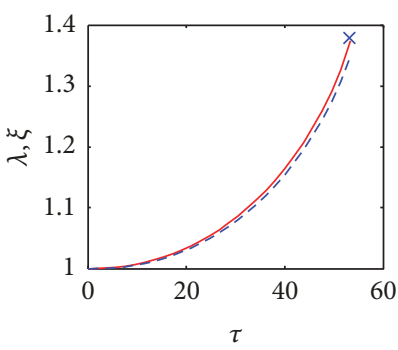

$\begin{array}{ll}- & \lambda \\ --- & \xi\end{array}$

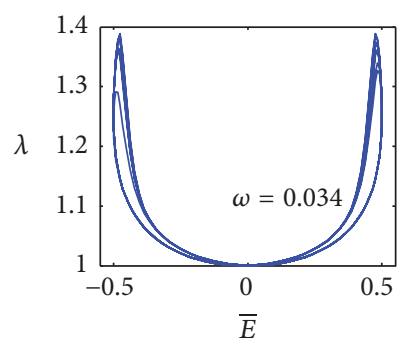

(h)

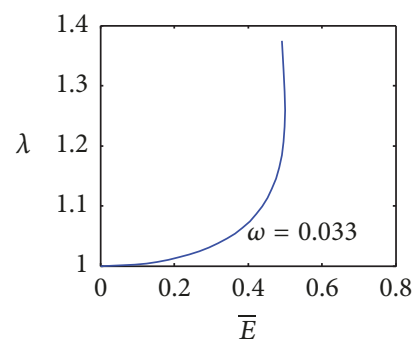

(k)

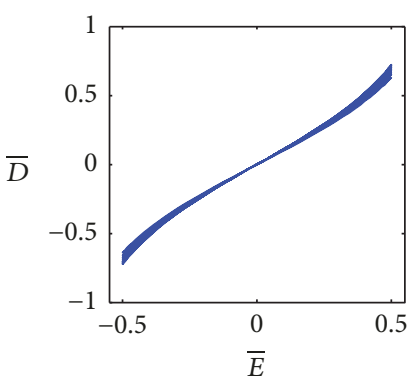

(c)

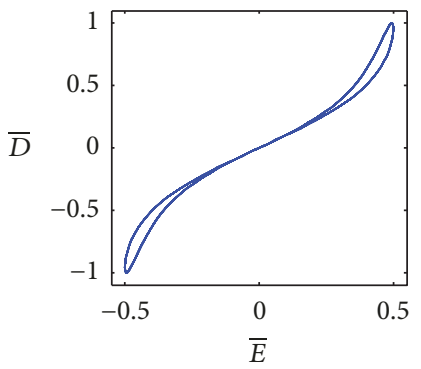

(f)

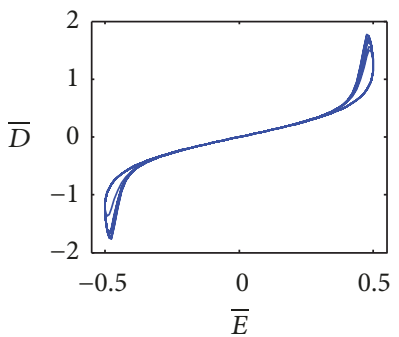

(i)

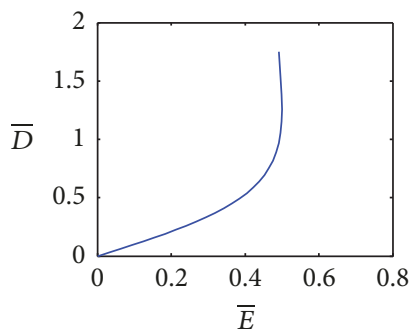

(1)

Figure 3: A membrane is subject to the applied cyclic nominal electric field. (a), (d), (g), and (j) are the calculated time-dependent total stretch and inelastic stretch. (b), (e), (h), and (k) are the stretch-electric field response. (c), (f), (i), and (l) are the dimensionless nominal electric displacement-nominal electric field curves. Four different dimensionless frequencies are used in the calculation with $\omega=10$ for $(\mathrm{a})$, (b), and (c), $\omega=1$ for (d), (e), and (f), $\omega=0.034$ for (g), (h), and (i), $\omega=0.033$ for (j), (k), and (l). 

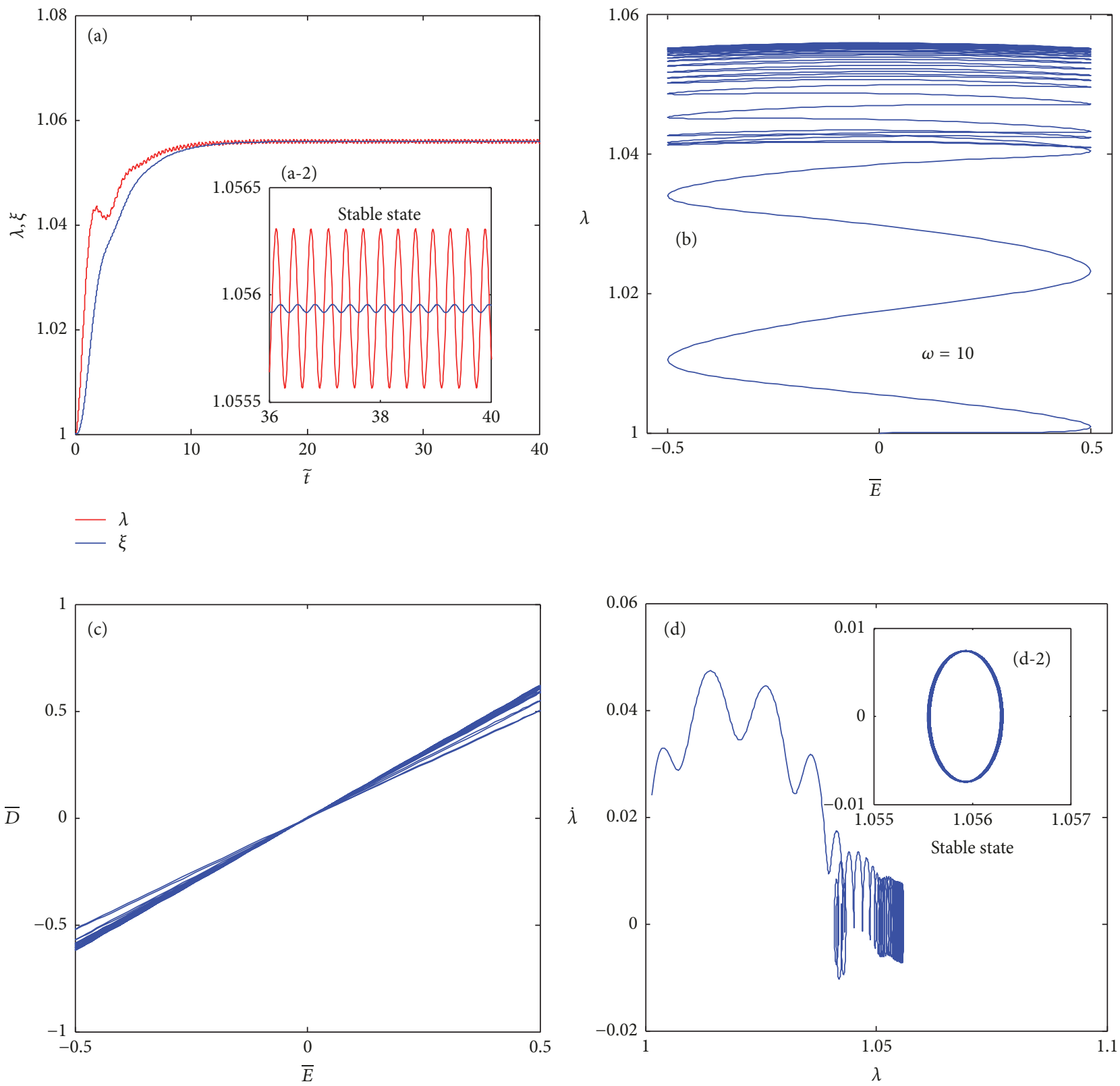

FIGURE 4: The total stretch $\lambda$ the viscous stretch $\xi$ (a), the nominal electric field-stretch response (b), the nominal electric field-nominal electric displacement (c), and the phase diagram (d) for the dimensionless frequency $\Omega=10$.

viscoelastic DE for high frequency. The phase diagram in Figure $4(\mathrm{~d})$ is not closed for $\Omega=10$ at the outset, emerging as a tangle of interlaced curves, which means that the system is aperiodic and unstable. However, the phase diagram in Figure 4(d-2) forms a closed loop after few cycles, implying a quasiperiodic vibration. The amplitude of the stretch $\lambda$ is extremely small; in other words, the quasiperiodic vibration is almost static.

Figure 5(a) shows the time-dependent behavior of the total stretch and the viscous stretch for $\Omega=11$. The stretch increases and stabilizes after few cycles. $\bar{E}-\lambda, \bar{E}-\bar{D}$ response and the phase diagram are plotted in Figures 5(b), 5(c), and 5(d). As can be seen from Figures 5(b) and 5(c), the hysteresis loop can be observed, indicating dissipation of energy. The phase paths in Figure 5(d) are presented in a closed loop when the system has achieved stable vibration after few cycles; this result shows that the system undergoes a nonlinear quasiperiodic oscillation.

The dynamic behaviors of the viscoelastic DE for $\Omega=0.1$ are shown in Figure 6 . When the DE is actuated by a relatively low frequency voltage for $\Omega=0.1$, the magnitude of the total stretch and the viscous stretch are both much larger and there will be significant dissipative energy during this cycle, as shown in Figures 6(a), 6(b), and 6(c), which is similar to the quasistatic response. The phase diagram is closed, indicating a steady state of oscillation, as indicated in Figure 6(d).

In addition, the critical value for the frequency to suppress the pull-in instability can be observed by solving (16). 


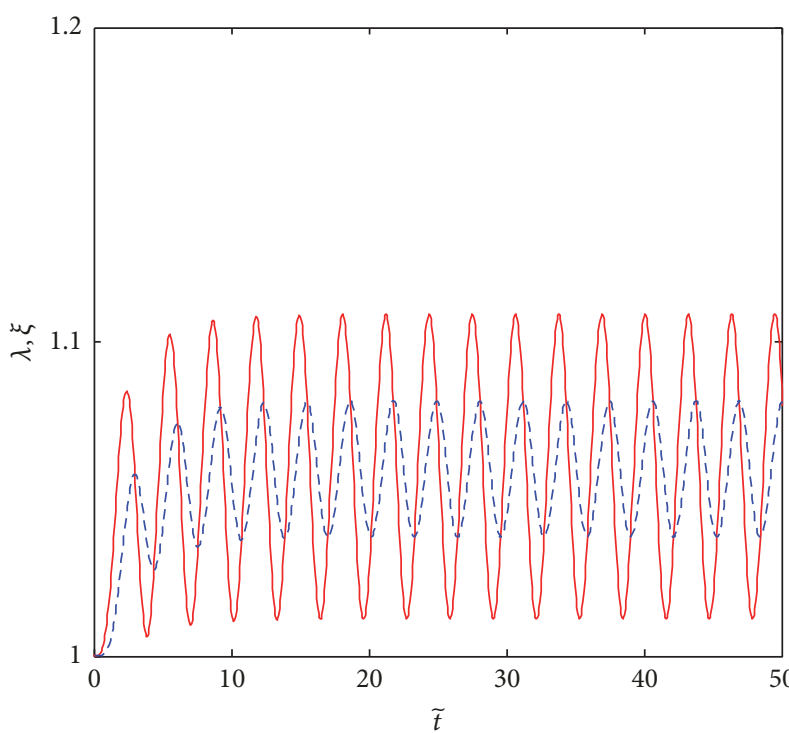

$-\lambda$

(a)

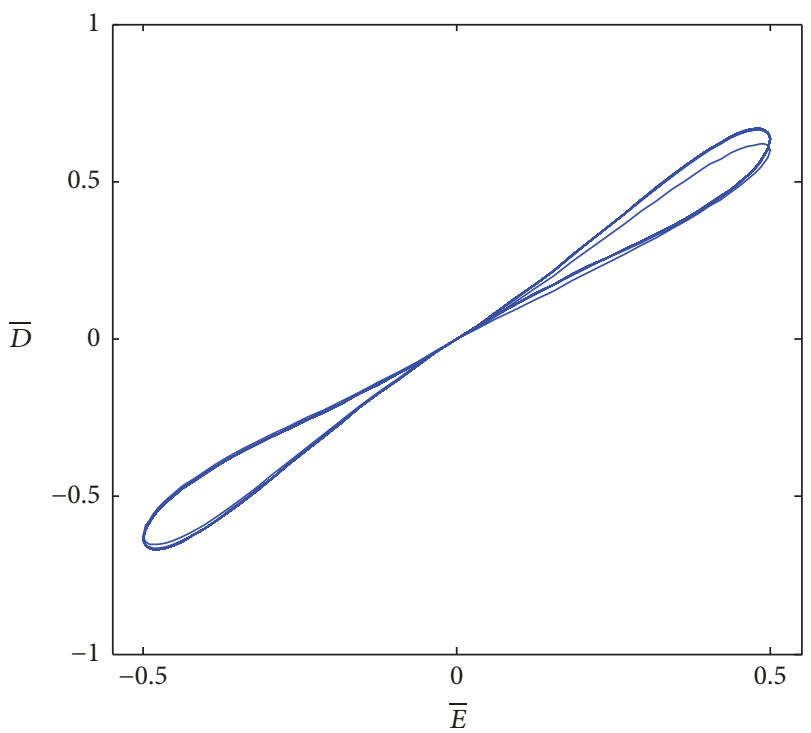

(c)

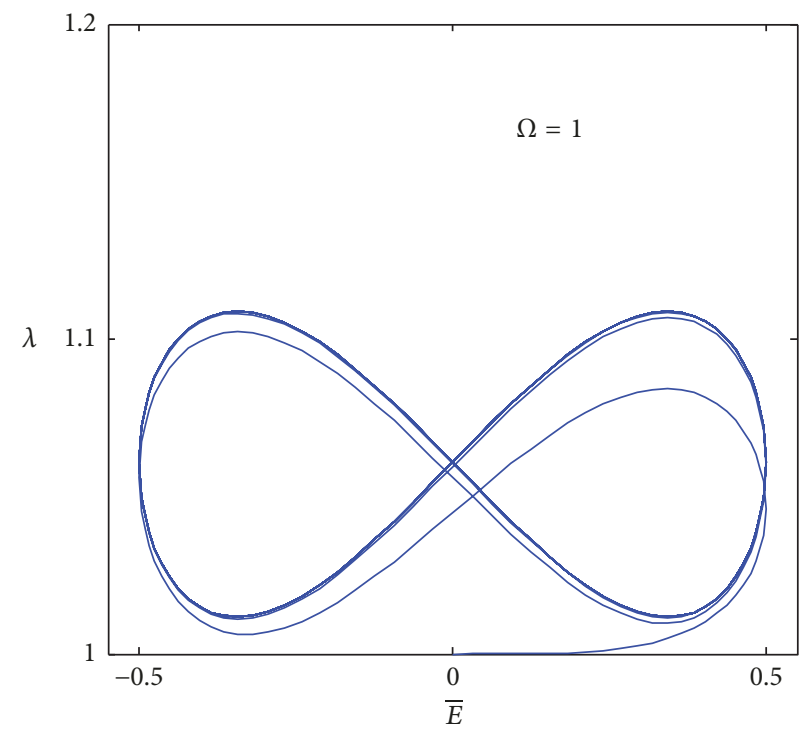

(b)

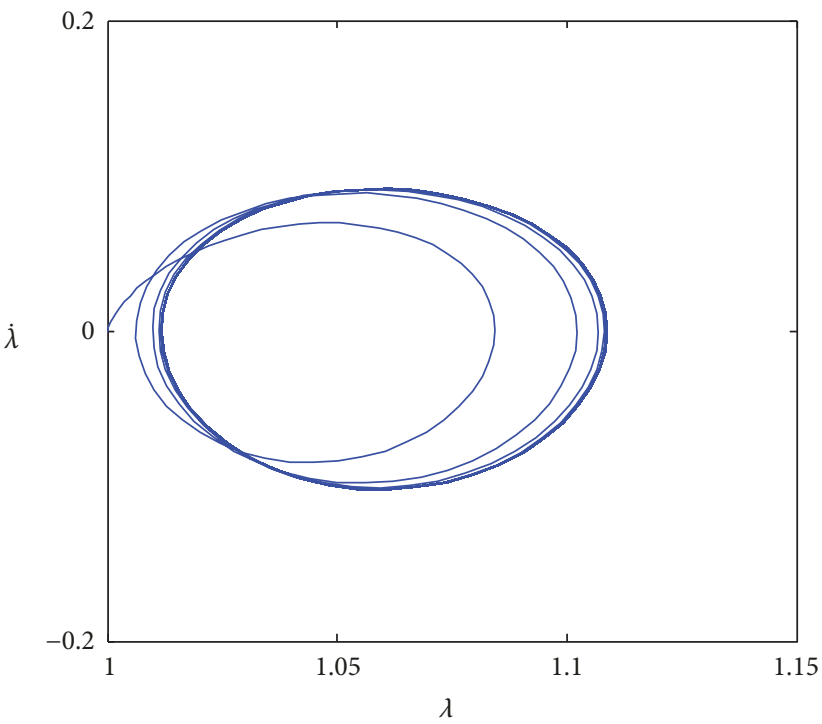

(d)

FIGURE 5: The total stretch $\lambda$, the viscous stretch $\xi$ (a), the nominal electric field-stretch response (b), the nominal electric field-nominal electric displacement (c), and the phase diagram (d) for the dimensionless frequency $\Omega=1$.

The critical value of the frequency is 0.0213 . The dynamic performances of the viscoelastic dielectric elastomer for very low frequency, $\Omega=0.0213$, are plotted in Figure 7. It can be seen that the stretch attains a maximum value which is about 1.5 than the critical value of quasistatic response. What is more, the hysteresis loop in the $\bar{E}-\lambda$ and $\bar{E}-\bar{D}$ plots becomes much bigger as compared with the high frequency in Figures 4-6 and the quasistatic response in Figure 3. That is to say, the dissipation of energy in the dynamic response is bigger than the quasistatic response. The reason is that the dynamic response of DE system under a cycle electric load is highly nonlinear and the asymmetry in the shape of the output cyclic deformation $\lambda(\widetilde{t})$ at low frequency for dynamic oscillation, and the nonlinear and asymmetry become more and more obviously, which would lead to more significant irreversible energy loss of the system in dynamic response. When the applied voltage frequency decrease to the critical value, for $\Omega \leq 0.0213$, the viscoelastic De will suffer the pullin instability after a certain time.

Poincaré map can be used to detect the stability transition of the system $[20,23,28]$. The Poincaré maps for the oscillation with the frequency $\Omega=10,1,0.1$, and 0.0213 are shown in Figure 8. It can be concluded that there exists strange attractor in the Poincare map for the oscillation with 

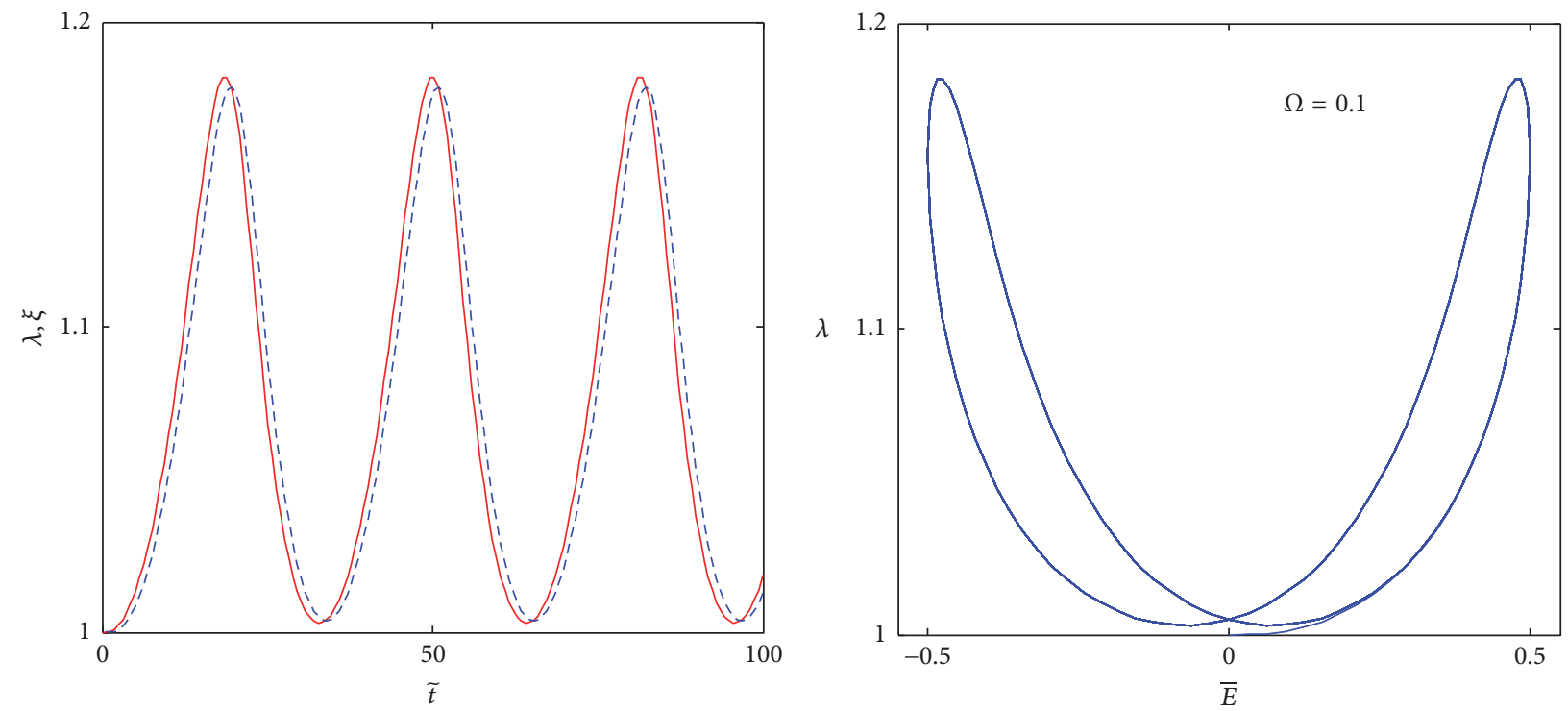

$-\lambda$

(a)

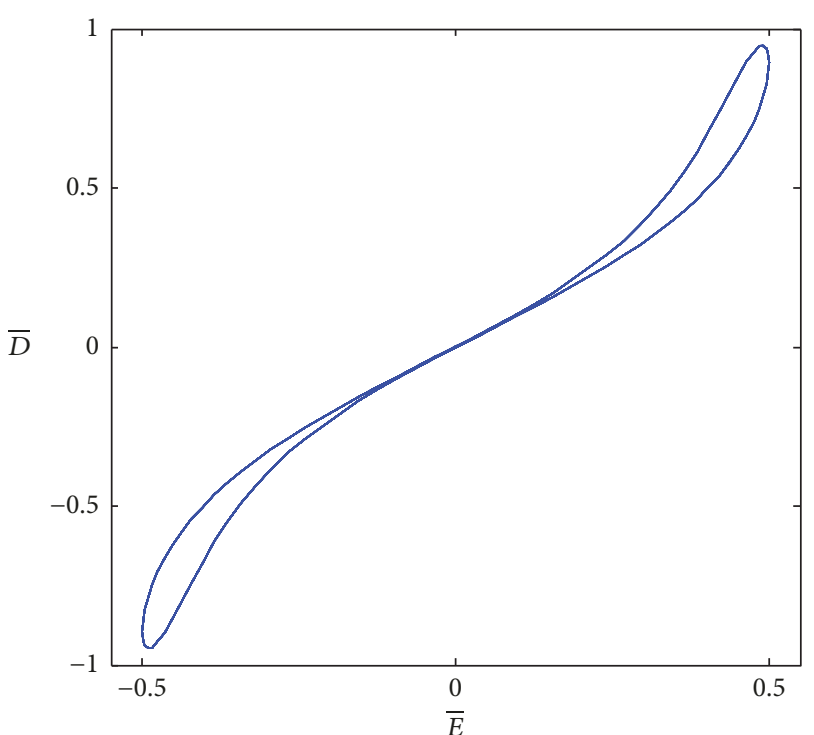

(c)

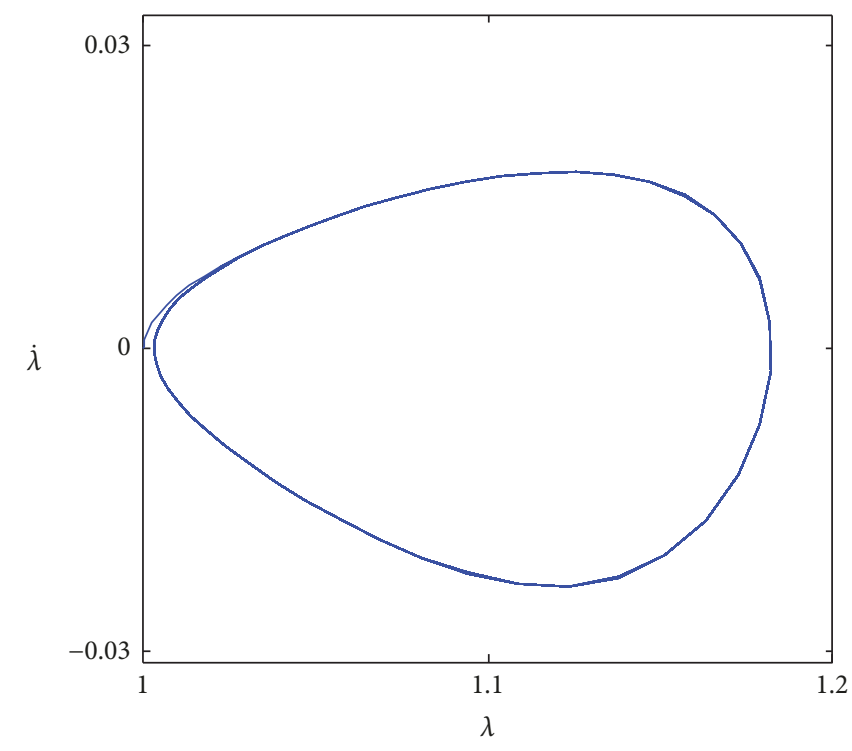

(d)

FIGURE 6: The total stretch $\lambda$, the viscous stretch $\xi$ (a), the nominal electric field-stretch response (b), the nominal electric field-nominal electric displacement (c), and the phase diagram (d) for the dimensionless frequency $\Omega=0.1$.

the frequency $\Omega=10$ in Figure 8(a) for the initiate cycles, which is the representative of an aperiodic vibration motion; with the creep of time, the oscillation of DE the Poincaré map at the frequency of $\Omega=10$ in Figure 8(a-2) shows one point, indicating single cycle oscillation. That is, the DE experiences a dynamic stability evolution from an aperiodic motion to the quasiperiodic motion for a relative high frequency. With the frequency decreasing, the Poincaré maps at the frequency of $\Omega=1,0.1$, and 0.0213 all have finite points, which mean that the motions are quasiperiodic and stable. As shown in Figure $8(\mathrm{~b})$, the motion of $\mathrm{DE}$ at $\Omega=1$ have two periods at least, compared to single cycle motion for frequencies 0.1 (Figure 8(c)) and 0.213 (Figure 8(d)).

3.3. Effect of Relaxation Time. What is more, the viscoelastic relaxation time of VHB ranges from hundreds of microseconds to hundreds of seconds $[39,40]$ and affects the creeping deformation $\xi$ of DE; that is, the enhancement of the prestretch (prestretch provides advantages to the electromechanical actuation to improve the electrical breakdown strength, avoiding instability and reducing the geometric thickness) followed by short relaxation approximately doubles that of 


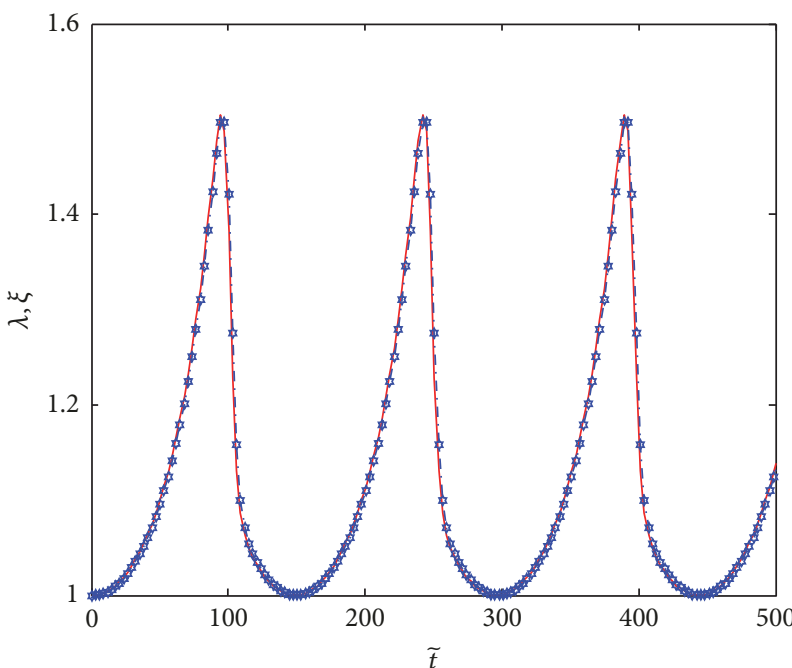

$-\lambda$

(a)

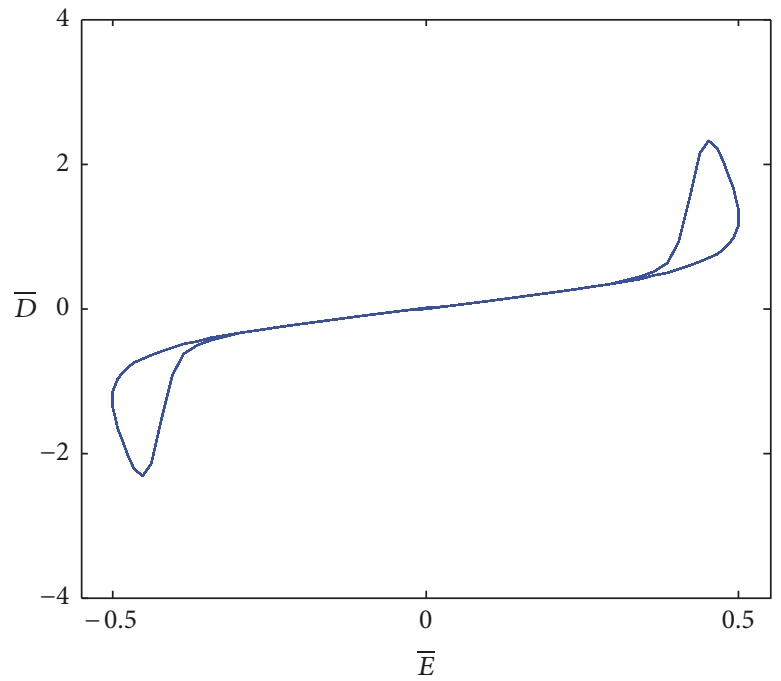

(c)

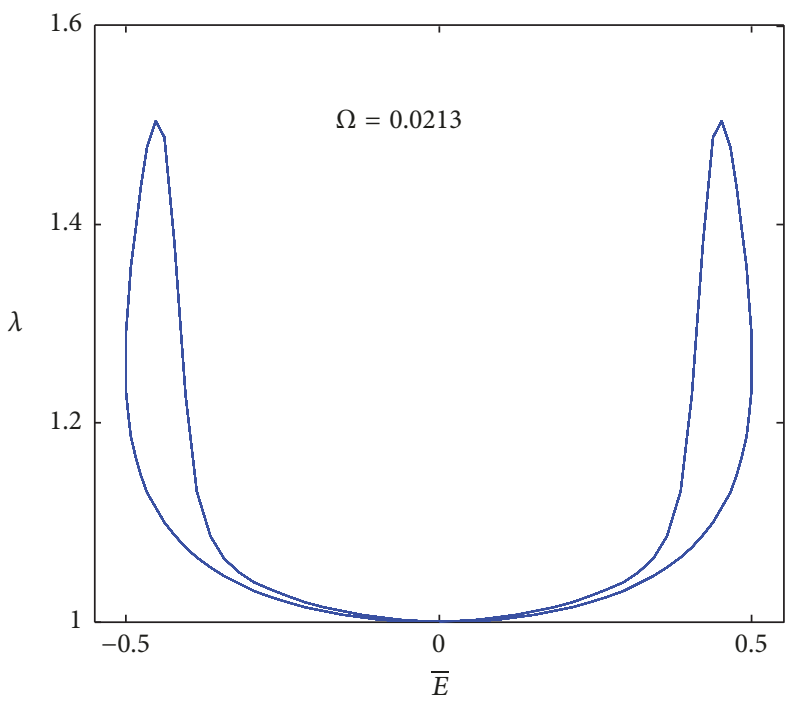

(b)

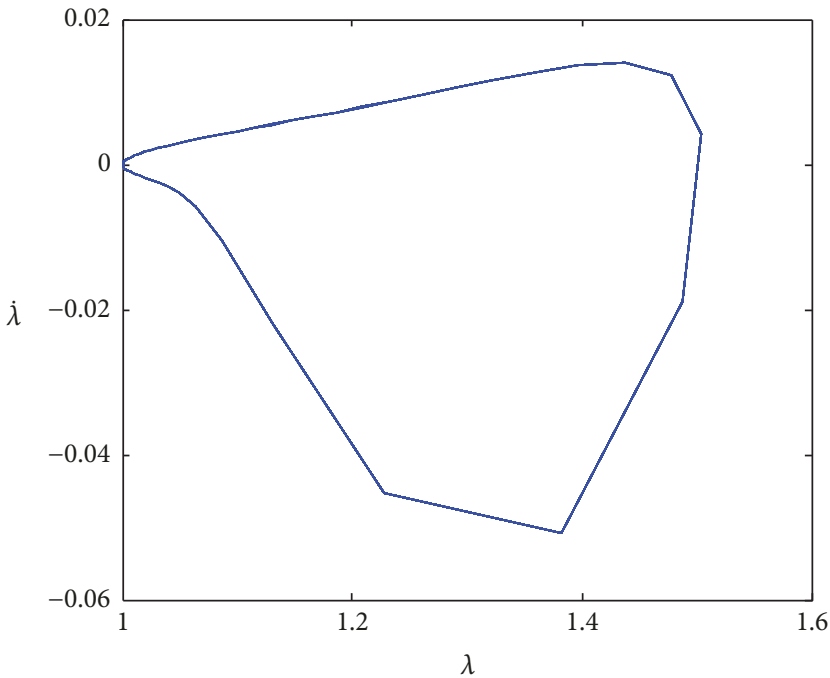

(d)

Figure 7: The total stretch $\lambda$, the viscous stretch $\xi$ (a), the nominal electric field-stretch response (b), the nominal electric field-nominal electric displacement (c), and the phase diagram (d) for the dimensionless frequency $\Omega=0.0213$.

the same prestretch but with long relaxation $[9,34]$. However, the relaxation time effects are seldom analyzed in the scope of viscoelasticity. When no prestress is applied $s=0$, we solve (16) under three levels of dimensionless viscoelastic relaxation time at the dimensionless frequency of $\Omega=$ 1 , with $\tilde{\tau}=1,10$, and 100, and plot the solution in Figure 9.

The oscillation of stretch $\lambda$ exhibits a strong nonlinearity with the coupled effects of relaxation times and frequencies. For a relative high relaxation time of $\widetilde{\tau}=10$ in Figure $9(\mathrm{~d})$, the stretch creeps with time and requires longer time to achieve a stable motion. For a higher relaxation time of $\tilde{\tau}=100$, the stretch of $\lambda$ will drift to a larger value at the stable state of oscillation after many cycles as shown in Figure 9(g-2).
It can also be observed that the creeping value of viscous stretch $(\xi)$ reduces gradually with the increasing value of relaxation times (Figures 9(a), 9(d), and 9(g)), and the creep can be almost eliminated in Figure $9(\mathrm{~g})$ when suitable values of relaxation times are applied. When the relaxation time increases, the viscous stretch becomes smaller, that is, because the viscoelastic deformation of the DE fails to keep up with electric field frequency leading to decreasing the viscous stretch.

As illustrated in Figure 9, the $\lambda$ - $\dot{\lambda}$ phase diagrams (Figures 9(b), 9(e), and 9(h)) are all presented in closed regions indicating that DE system experiences a nonlinear quasiperiodic oscillation and the dynamic oscillation of the DE system is stable. When the relaxation time increases from $\widetilde{\tau}=1$ to 

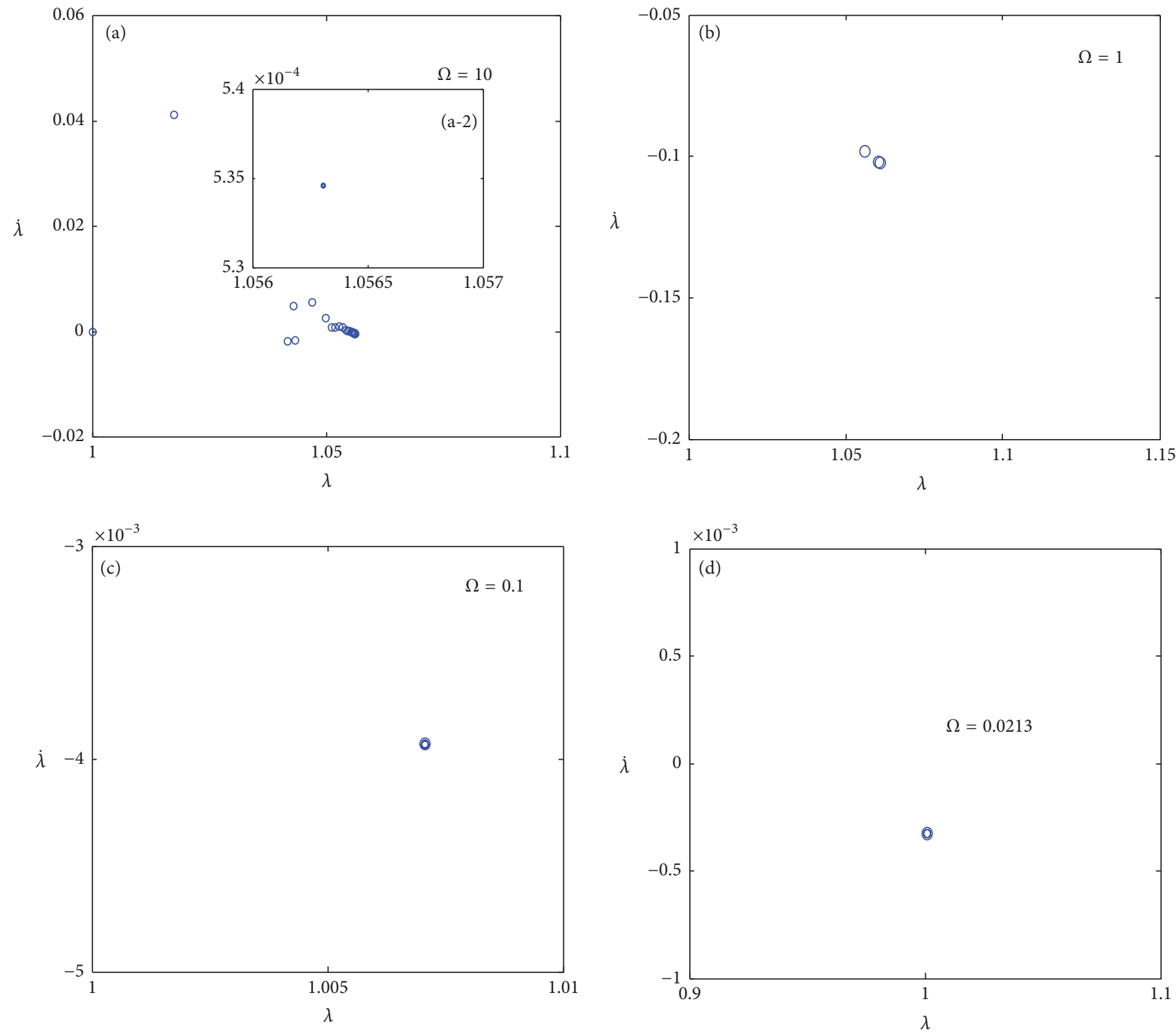

Figure 8: Poincaré map for the oscillation under a cyclic electric load with different dimensionless frequencies (a) $\Omega=10$, (b) $\Omega=1$, (c) $\Omega=0.1$, and (d) $\Omega=0.0213$.

$\tilde{\tau}=100$, the Poincaré maps (Figures 9(c), 9(f), and 9(i)) all show finite points with 2,3 , and 8 , respectively. That is to say, the DE system may experiences a stability evolution from a single cycle motion to multicycle motion with the increasing relaxation times for a fixed frequency.

\section{Conclusions}

Although the dynamics of DE are widely studied recently, this study gives a further understanding of the effect of viscoelasticity and frequency on dynamic response of $\mathrm{DE}$ and can help to guide the design of dynamic and static applications in DE actuators.

The dynamic behavior of a viscoelastic DE under a cycle electric field is presented to compare with the quasistatic behavior. The time-dependent dynamic deformation, the hysteresis process, and the dynamic stability undergoing viscoelastic dissipative processes are investigated by using the theory of nonequilibrium thermodynamics. The results show that when the frequency of the applied voltage decreases, whether the quasistatic or the dynamic performance, the total stretch and the viscous stretch both become larger. In addition, the energy dissipated per cycle increases with the decreasing frequency and there exists a critical value of the frequency at which the pull-in instability takes place. When the applied voltage frequency is below a critical value, the viscoelastic DE cannot attain a steady state of oscillation and may have instability. The system of the viscoelastic DE also presents stability transition from an aperiodic motion to the quasiperiodic motion for a relative high frequency and experiences a stability evolution from single cycle motion to 

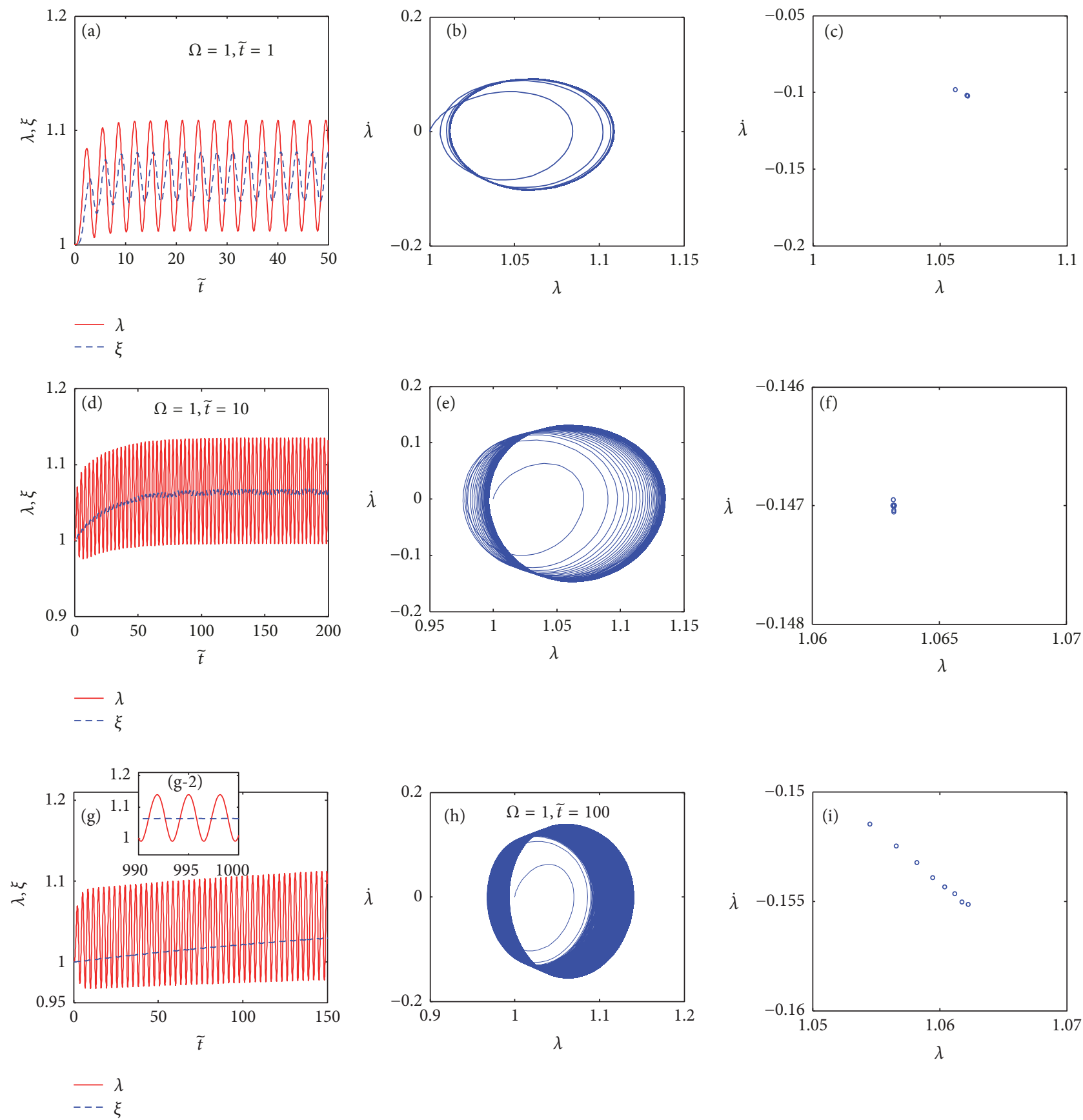

FIGURE 9: The total stretches $\lambda$, the viscous stretches $\xi((\mathrm{a}),(\mathrm{d})$, and $(\mathrm{g}))$, the phase diagrams $((\mathrm{c}),(\mathrm{e})$, and $(\mathrm{h}))$, and the Poincare maps $((\mathrm{c})$, (f), and (i)) at dimensionless frequency of $\Omega=1$ for different dimensionless relaxation times $\tilde{\tau}=1,10$, and 100 .

multicycle motion with the increasing relaxation times for a fixed frequency. The creeping value of viscous stretch of the dashpot reduces gradually with the increasing value of relaxation times and the creep can be almost eliminated at a suitable value of relaxation time.

\section{Conflicts of Interest}

The authors declare that they have no conflicts of interest.

\section{Acknowledgments}

This research was supported by the National Natural Science Foundation of China (Grant no. 11402246). The authors gratefully acknowledge these supports.

\section{References}

[1] J. W. Kwak, H. J. Chi, K. M. Jung et al., "A face robot actuated with artificial muscle based on dielectric elastomer," Journal of 
Mechanical Science and Technology, vol. 19, no. 2, pp. 578-588, 2005.

[2] G. Kovacs, L. Düring, S. Michel, and G. Terrasi, "Stacked dielectric elastomer actuator for tensile force transmission," Sensors and Actuators A: Physical, vol. 155, no. 2, pp. 299-307, 2009.

[3] C. Keplinger, M. Kaltenbrunner, N. Arnold, and S. Bauer, "Röntgen's electrode-free elastomer actuators without electromechanical pull-in instability," Proceedings of the National Acadamy of Sciences of the United States of America, vol. 107, no. 10, pp. 4505-4510, 2010.

[4] T. McKay, B. O’Brien, E. Calius, and I. Anderson, "Self-priming dielectric elastomer generators," Smart Materials and Structures, vol. 19, no. 5, Article ID 055025, 2010.

[5] F. Carpi, D. De Rossi, R. Kornbluh, R. Pelrine, and P. SommerLarsen, "Dielectric elastomers as electromechanical transducers: Fundamentals, Materials, Devices, Models and Applications of an Emerging Electroactive Polymer Technology," Dielectric Elastomers as Electromechanical Transducers, p. 329, 2008.

[6] P. Brochu and Q. Pei, "Advances in dielectric elastomers for actuators and artificial muscles," Macromolecular Rapid Communications, vol. 31, no. 1, pp. 10-36, 2010.

[7] R. K. Sahu, A. Saini, D. Ahmad, K. Patra, and J. Szpunar, "Estimation and validation of maxwell stress of planar dielectric elastomer actuators," Journal of Mechanical Science and Technology, vol. 30, no. 1, pp. 429-436, 2016.

[8] L. Liu, J. Li, Y. Liu, J. Leng, J. Zhao, and J. Zhao, "Electric field induced variation of temperature and entropy in dielectric elastomers," Journal of Mechanical Science and Technology, vol. 29, no. 1, pp. 109-114, 2015.

[9] J.-S. Plante and S. Dubowsky, "Large-scale failure modes of dielectric elastomer actuators," International Journal of Solids and Structures, vol. 43, no. 25-26, pp. 7727-7751, 2006.

[10] J.-S. Plante and S. Dubowsky, "On the performance mechanisms of Dielectric Elastomer Actuators," Sensors and Actuators A: Physical, vol. 137, no. 1, pp. 96-109, 2007.

[11] M. Molberg, Y. Leterrier, C. J. G. Plummer et al., "Frequency dependent dielectric and mechanical behavior of elastomers for actuator applications," Journal of Applied Physics, vol. 106, no. 5, Article ID 054112, 2009.

[12] F. Carpi, S. Bauer, and D. De Rossi, "Stretching dielectric elastomer performance," Science, vol. 330, no. 6012, pp. 1759$1761,2010$.

[13] P. Dubois, S. Rosset, M. Niklaus, M. Dadras, and H. Shea, "Voltage control of the resonance frequency of dielectric electroactive polymer (DEAP) membranes," Journal of Microelectromechanical Systems, vol. 17, no. 5, pp. 1072-1081, 2008.

[14] J. W. Fox and N. C. Goulbourne, "On the dynamic electromechanical loading of dielectric elastomer membranes," Journal of the Mechanics and Physics of Solids, vol. 56, no. 8, pp. 2669-2686, 2008.

[15] K. Hochradel, S. J. Rupitsch, A. Sutor, R. Lerch, D. K. Vu, and P. Steinmann, "Dynamic performance of dielectric elastomers utilized as acoustic actuators," Applied Physics A: Materials Science \& Processing, vol. 107, no. 3, pp. 531-538, 2012.

[16] J. Zhu, S. Cai, and Z. Suo, "Nonlinear oscillation of a dielectric elastomer balloon," Polymer International, vol. 59, no. 3, pp. 378$383,2010$.

[17] J. Zhu, S. Cai, and Z. Suo, "Resonant behavior of a membrane of a dielectric elastomer," International Journal of Solids and Structures, vol. 47, no. 24, pp. 3254-3262, 2010.
[18] H. Yong, X. He, and Y. Zhou, "Dynamics of a thick-walled dielectric elastomer spherical shell," International Journal of Engineering Science, vol. 49, no. 8, pp. 792-800, 2011.

[19] T. Li, S. Qu, and W. Yang, "Electromechanical and dynamic analyses of tunable dielectric elastomer resonator," International Journal of Solids and Structures, vol. 49, no. 26, pp. 3754-3761, 2012.

[20] B.-X. Xu, R. Mueller, A. Theis, M. Klassen, and D. Gross, "Dynamic analysis of dielectric elastomer actuators," Applied Physics Letters, vol. 100, no. 11, Article ID 112903, 2012.

[21] K. Jia, T. Lu, and T. J. Wang, "Response time and dynamic range for a dielectric elastomer actuator," Sensors and Actuators A: Physical, vol. 239, pp. 8-17, 2016.

[22] B. Li, J. Zhang, L. Liu, H. Chen, S. Jia, and D. Li, "Modeling of dielectric elastomer as electromechanical resonator," Journal of Applied Physics, vol. 116, no. 12, Article ID 124509, 2014.

[23] J. Sheng, H. Chen, B. Li, and Y. Wang, "Nonlinear dynamic characteristics of a dielectric elastomer membrane undergoing in-plane deformation," Smart Materials and Structures, vol. 23, no. 4, Article ID 045010, 2014.

[24] F. Wang, T. Lu, and T. J. Wang, "Nonlinear vibration of dielectric elastomer incorporating strain stiffening," International Journal of Solids and Structures, vol. 87, pp. 70-80, 2016.

[25] A. York, J. Dunn, and S. Seelecke, "Experimental characterization of the hysteretic and rate-dependent electromechanical behavior of dielectric electro-active polymer actuators," Smart Materials and Structures, vol. 19, no. 9, Article ID 094014, 2010.

[26] P. Lochmatter, G. Kovacs, and M. Wissler, "Characterization of dielectric elastomer actuators based on a visco-hyperelastic film model," Smart Materials and Structures, vol. 16, no. 2, article no. 028, pp. 477-486, 2007.

[27] C. Keplinger, M. Kaltenbrunner, N. Arnold, and S. Bauer, "Capacitive extensometry for transient strain analysis of dielectric elastomer actuators," Applied Physics Letters, vol. 92, no. 19, Article ID 192903, 2008.

[28] J. Zhang, H. Chen, B. Li, D. McCoul, and Q. Pei, "Coupled nonlinear oscillation and stability evolution of viscoelastic dielectric elastomers," Soft Matter, vol. 11, no. 38, pp. 7483-7493, 2015.

[29] J. Zhang, L. Tang, B. Li, Y. Wang, and H. Chen, "Modeling of the dynamic characteristic of viscoelastic dielectric elastomer actuators subject to different conditions of mechanical load," Journal of Applied Physics, vol. 117, no. 8, Article ID 084902, 2015.

[30] J. Zhang, J. Zhao, H. Chen, and D. Li, "Dynamic analyses of viscoelastic dielectric elastomers incorporating viscous damping effect," Smart Materials \& Structures, vol. 26, no. 1, Article ID 015010, 2017.

[31] Y. Wang, H. Xue, H. Chen, and J. Qiang, "A dynamic viscohyperelastic model of dielectric elastomers and their energy dissipation characteristics," Applied Physics A: Materials Science \& Processing, vol. 112, no. 2, pp. 339-347, 2013.

[32] C. Zhang, H. Chen, B. Li, Y. Wang, L. Liu, and D. Li, "Investigation on static and dynamic performance of a hinge configuration with integrated dielectric elastomers," Journal of Applied Polymer Science, vol. 132, no. 11, p. 41630, 2015.

[33] J. Sheng, H. Chen, L. Liu, J. Zhang, Y. Wang, and S. Jia, "Dynamic electromechanical performance of viscoelastic dielectric elastomers," Journal of Applied Physics, vol. 114, no. 13, Article ID 134101, 2013.

[34] W. Hong, "Modeling viscoelastic dielectrics," Journal of the Mechanics and Physics of Solids, vol. 59, no. 3, pp. 637-650, 2011. 
[35] X. Zhao, S. J. A. Koh, and Z. Suo, "Nonequilibrium thermodynamics of dielectric elastomers," International Journal of Applied Mechanics, vol. 3, no. 2, pp. 203-217, 2011.

[36] C. Chiang Foo, S. Cai, S. Jin Adrian Koh, S. Bauer, and Z. Suo, "Model of dissipative dielectric elastomers," Journal of Applied Physics, vol. 111, no. 3, Article ID 034102, 2012.

[37] J. Huang, T. Li, C. Chiang Foo, J. Zhu, D. R. Clarke, and Z. Suo, "Giant, voltage-actuated deformation of a dielectric elastomer under dead load," Applied Physics Letters, vol. 100, no. 4, Article ID 041911, 2012.

[38] A. N. A. Gent, "A new constitutive ralation for rubber," Rubber Chemistry Technology, vol. 69, no. 1, pp. 59-61, 1996.

[39] M. Wissler and E. Mazza, "Mechanical behavior of an acrylic elastomer used in dielectric elastomer actuators," Sensors and Actuators A: Physical, vol. 134, no. 2, pp. 494-504, 2007.

[40] S. Michel, X. Q. Zhang, M. Wissler, C. Löwe, and G. Kovacs, "A comparison between silicone and acrylic elastomers as dielectric materials in electroactive polymer actuators," Polymer International, vol. 59, no. 3, pp. 391-399, 2010. 


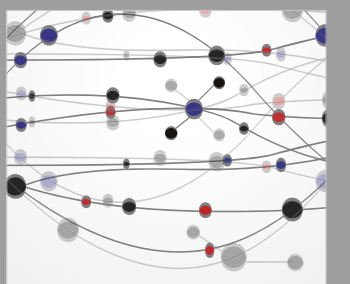

The Scientific World Journal
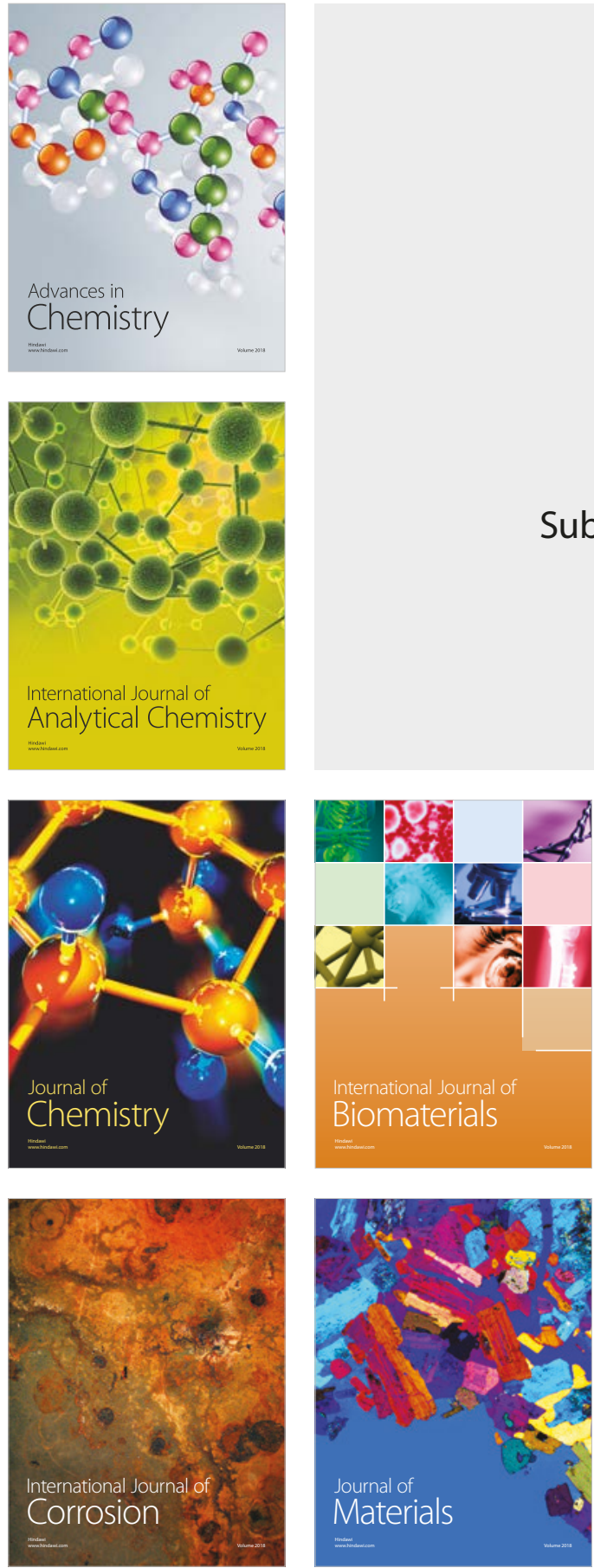

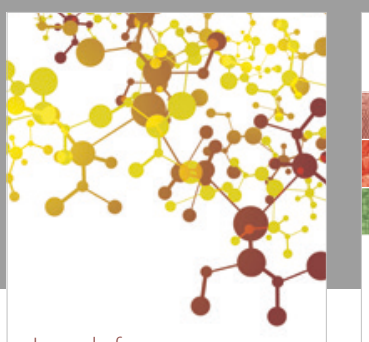

Journal of

Applied Chemistry
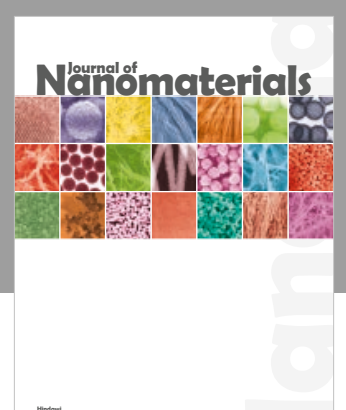

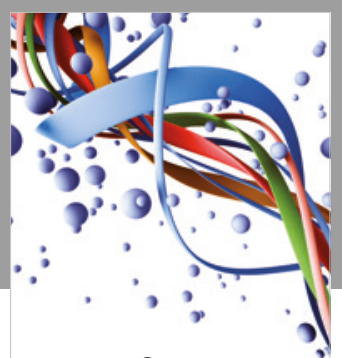

Scientifica

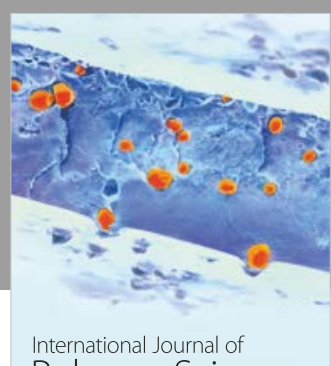

Polymer Science

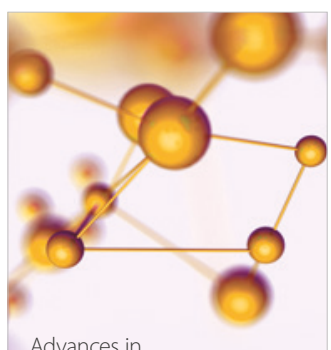

Physical Chemistry
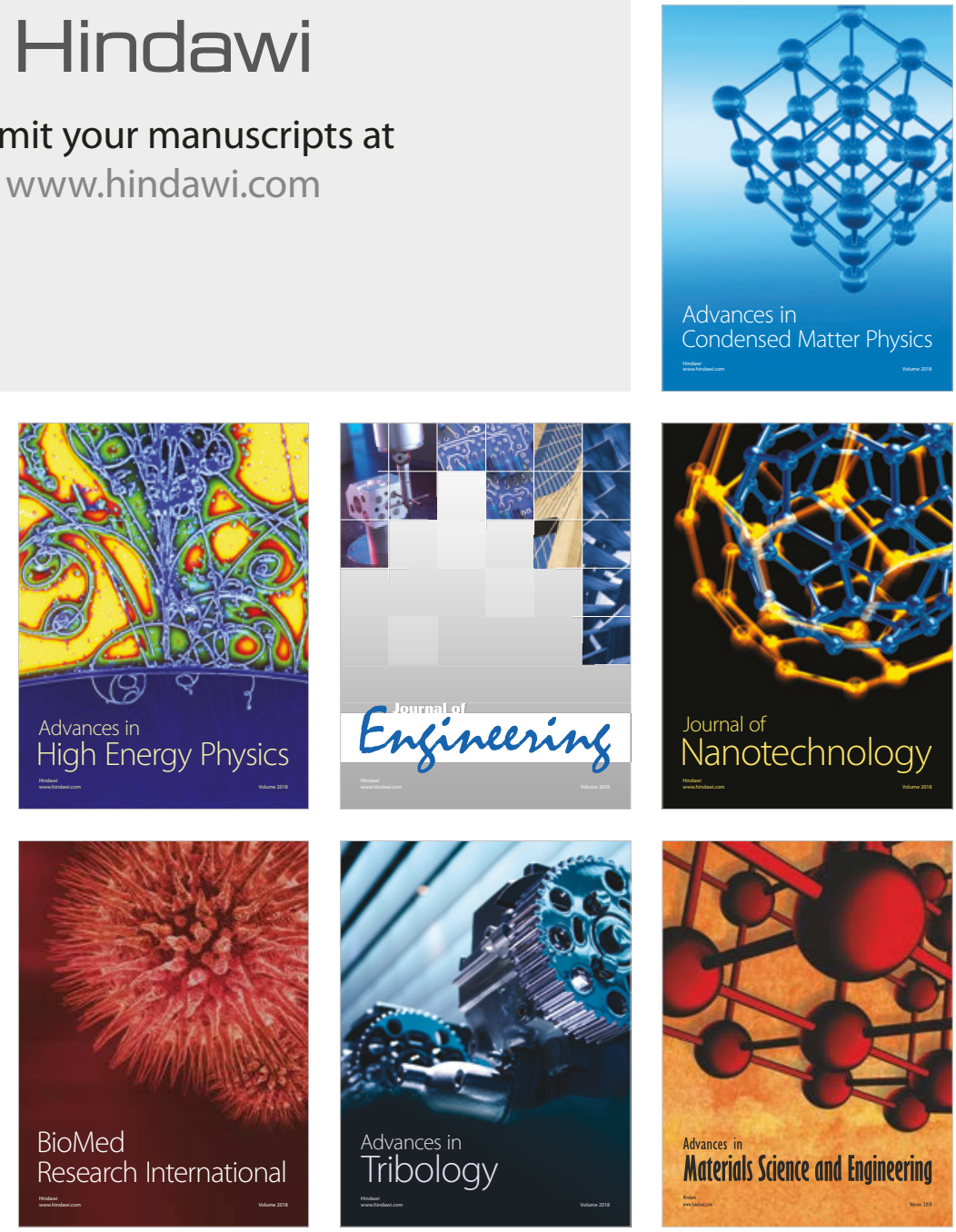